\title{
Nonlinear analysis of doubly curved shells: An analytical approach
}

\author{
Y NATH and K SANDEEP \\ Applied Mechanics Department, Indian Institute of Technology Delhi, New \\ Delhi 110016, India \\ ${ }^{2}$ Birla Institute of Technology, Pilani 333031 , India \\ e-mail: ynath@am.iitd.ernet.in
}

\begin{abstract}
Dynamic analogues of von Karman-Donnell type shell equations for doubly curved, thin isotropic shells in rectangular planform are formulated and expressed in displacement components. These nonlinear partial differential equations of motion are linearized by using a quadratic extrapolation technique. The spatial and temporal discretization of differential equations have been carried out by finite-degree Chebyshev polynomials and implicit Houbolt timemarching techniques respectively. Multiple regression based on the least square error norm is employed to eliminate the incompatability generated due to spatial discretization (equations $>$ unknowns). Spatial convergence study revealed that nine term expansion of each displacement in $x$ and $y$ respectively, is sufficient to yield fairly accurate results. Clamped and simply supported immovable doubly curved shallow shells are analysed. Results have been compared with those obtained by other numerical methods. Considering uniformly distributed normal loading, the results of static and dynamic analyses are presented.
\end{abstract}

Keywords. Nonlinear mechanics; doubly curved shells; analytical solutions; Chebyshev technique.

\section{Introduction}

The superiority of numerical methods like finite element, finite difference and boundary element methods for analysis of structural elements having complex geometrical configuration and loading is well-established. The involved computational programming and cost however, often prohibits their frequent use in problems of shells having rectangular planform boundaries. The most commonly employed analytical approach in the rectangular domain is based on Fourier series approximation to spatial functions (Chia 1980; Khedir \& Reddy 1989). It is well-known that Fourier series have poor convergence. Therefore, there is a time-honoured need to have an efficient analytical methodology of solution, which could serve as a bench-mark solution for the numerical methods. Chebyshev polynomials are orthogonal functions and have the property of minimax (Fox \& Parker 1968). The

A list of symbols is given at the end of the paper 
convergence and accuracy of the Chebyshev series solution to axisymmetric plate and shell problems have been investigated by Alwar \& Nath $(1976,1978)$. The solution of linear biharmonic equation in rectangular domain by the Chebyshev series is given by Evans \& Murphy (1981). Nath \& Sandeep (1995) have investigated the nonlinear dynamic response of rectangular plates using double Chebyshev series.

In the present investigation, the methodology based on the Chebyshev series approximation to functions in space domain is developed for nonlinear static and dynamic analyses of doubly curved shells. The spatial descretization of rectangular domain by Chebyshev series gives rise to more algebraic equations than the number of unknown coefficients in the series depending upon the domain conditions. In order to ensure the uniqueness of the solution, the technique of multiple linear regression is employed. The nonlinear terms are approximated at each step of marching variable by their values at three preceding steps. Velocity and acceleration terms are evaluated by implicit Houbolt time-marching scheme (Houbolt 1950). Convergence study has been carried out and it is found that nine term expansion of the Chebyshev series to each function in $x$ and $y$, respectively is sufficient to yield accurate results. Clamped and simply supported cylindrical panels and doubly curved shells under uniform static and dynamic loading are analysed. The results have been compared with those obtained by other numerical methods.

\section{Field equations}

The geometry and coordinate system are shown in figure 1. Neglecting the in-plane and rotatory inertia, governing equations of motion for the doubly curved shells undergoing moderately large deflections are expressed in non-dimensional form (Nath et al 1987) as:

$$
\begin{aligned}
& w_{x x x x}+2 \xi^{2} w_{x x y y}+\xi^{4} w_{y y y y}-12\left(w_{x x}+R_{1}\right)\left[u_{x}+\xi \nu v_{y}+0.5\left(w_{x}\right)^{2}\right. \\
& \left.\quad+0.5 \xi^{2} \nu\left(w_{y}\right)^{2}-R_{1} w-\nu R_{2} w\right]-12\left(\xi^{2} w_{y y}+R_{2}\right)\left[\xi v_{y}+\nu u_{x}\right. \\
& \left.\quad+0.5 \nu\left(w_{x}\right)^{2}+0.5 \xi^{2}\left(w_{y}\right)^{2}-R_{2} w-\nu R_{1} w\right] \\
& \quad-12(1-\nu) \xi\left[\xi u_{y}+v_{x}+\xi w_{x} w_{y}\right] w_{x y}-q+w_{t t}+c w_{t}=0
\end{aligned}
$$

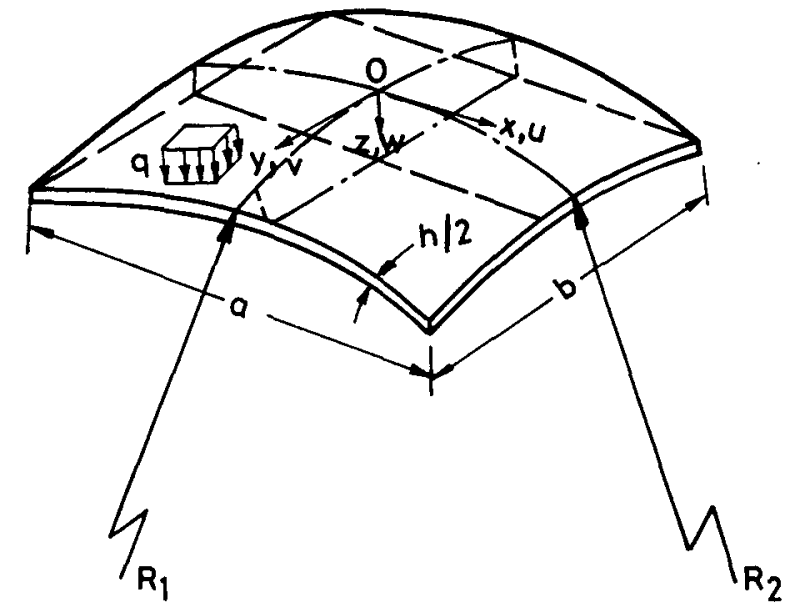

Figure 1. Geometry of shell. 


$$
\begin{aligned}
& u_{x x}+\xi^{2} d_{1} u_{y y}+\xi d_{2} v_{x y}+w_{x}\left[w_{x x}+\xi^{2} d_{1} w_{y y}\right]+\xi^{2} d_{2} w_{y} w_{x y} \\
& \quad-\left(R_{1}+\nu R_{2}\right) w_{x}=0 \\
& \xi^{2} v_{y y}+d_{1} v_{x x}+\xi d_{2} u_{x y}+\xi w_{y}\left[d_{1} w_{x x}+\xi^{2} w_{y y}\right]+\xi d_{2} w_{x} w_{x y} \\
& \quad-\left(R_{2}+\nu R_{1}\right) w_{y}=0
\end{aligned}
$$

where the subscript denotes the partial derivative with respect to the following suffix. The non-dimensional quantities are defined by

$$
\begin{aligned}
w & =w^{*} / h, x=x^{*} / a, y=y^{*} / b, \xi=a / b, d_{1}=(1-\nu) / 2, d_{2}=(1+\nu) / 2, \\
u & =a u^{*} / h^{2}, v=a v^{*} / h^{2}, q=q^{*} a^{4} /(D h), R_{1}=R_{1}^{*} b^{2} / h, R_{2}=R_{2}^{*} b^{2} / h, \\
t & =t^{*}\left[D /\left(\gamma a^{4} h\right)\right]^{0.5}, c=c^{*}\left[\left(\gamma a^{4} h\right) / D\right]^{0.5}, D=E h^{3} / 12\left(1-\nu^{2}\right) .
\end{aligned}
$$

The boundary conditions are considered as follows.

(1) For all four edges simply supported and immovably constrained against in-plane translation,

$$
\begin{aligned}
& y=1 \&-1 ; \quad u=v=w=0, \quad \xi^{2} w_{y y}+\nu w_{x x}=0 . \\
& x=1 \&-1 ; \quad u=v=w=0, \quad w_{x x}+\xi^{2} \nu w_{y y}=0 .
\end{aligned}
$$

(2) For all four edges clamped and immovably constrained against in-plane translation,

$$
\begin{aligned}
& y=1 \&-1 ; \quad u=v=w=0, \quad w_{y}=0, \\
& x=1 \&-1 ; \quad u=v=w=0, \quad w_{x}=0 .
\end{aligned}
$$

The initial conditions are: $t=0 ; w=0, w_{t}=0$.

\section{Method of solution}

The displacement fields $u, v, w$ and loading $q$ can be approximated in space domain finite degree Chebyshev polynomials (Fox \& Parker 1968) as

$$
(u, v, w, q)=\delta \sum_{I=0}^{M} \sum_{j=0}^{N}\left(u_{i, j}, v_{i, j}, w_{i, j}, q_{i, j}\right) T_{j}(x) T_{j}(y),
$$

where

$$
\begin{array}{ll}
\delta=1 / 4, & \text { if } i=0, \quad \text { and } j=0, \\
\delta=1 / 2, & \text { if } i=0, \quad \text { and } j \neq 0, \quad \text { or } i \neq 0 \quad \text { and } j=0 \\
\delta=1, & \text { otherwise. }
\end{array}
$$

The spatial derivative of $\phi(x, y, t)$ can be expressed as

$$
\partial^{(r s)} \phi / \partial x^{r} \partial y^{s}=\delta \sum_{i=0}^{M-r} \sum_{j=0}^{N-s} \phi_{i, j}^{(r s)} T_{i}(x) T_{j}(y)
$$


where $r$ and $s$ are the order of derivatives with respect to $x$ and $y$ respectively. The derivative functions $\phi_{i, j}^{r s}$ can be evaluated by using the recurrence relations

$$
\begin{aligned}
& \phi_{i, j}^{(r-1) s}=\left[\phi_{i-1, j}^{r s}+\phi_{i+1, j}^{r s}\right] / 2 i, \\
& \phi_{i, j}^{r(s-1)}=\left[\phi_{i, j-1}^{r s}+\phi_{i, j+1}^{r s}\right] / 2 j .
\end{aligned}
$$

Nonlinearity in the governing equations of motion is due to the product of the dependent variables. The quadratic extrapolation technique is used for the linearization and a typical nonlinear function $G_{j}$ can be expressed at any step $j$ as,

$$
\begin{aligned}
G_{j} & =\left(\phi^{r}\right)_{J}\left(\phi^{s}\right)_{J} \\
& =\left[\delta \sum_{i=0}^{M-r} \sum_{j=0}^{N} \phi_{i, j}^{r} T_{i}(x) T_{j}(y)\right]_{J}\left[\delta \sum_{i=0}^{M} \sum_{j=0}^{N-s} \phi_{i, j}^{s} T_{i}(x) T_{j}(y)\right]_{J},
\end{aligned}
$$

where,

$$
\left(\phi_{i, j}\right)_{J}=A\left(\phi_{i, j}\right)_{J-1}+B\left(\phi_{i, j}\right)_{J-2}+C\left(\phi_{i, j}\right)_{J-3} .
$$

During the initial steps of marching variable, the coefficients $A, B, C$ of the quadratic extrapolation scheme of linearization take the following values

$$
1,0,0(J=1) ; \quad 2,-1,0(J=2) ; \quad 3,-3,1(J>3) \text {. }
$$

The product of two double Chebyshev polynomials can be expressed as

$$
\begin{aligned}
T_{i}(x) T_{j}(y) T_{k}(x) T_{p}(y)= & {\left[T_{i+k}(x) T_{j+p}(y)+T_{i+k}(x) T_{j-p}(y)\right.} \\
& \left.+T_{i-k}(x) T_{j+p}(y)+T_{i-k}(x) T_{j-p}(y)\right] / 4 .
\end{aligned}
$$

Implicit Houbolt time-marching method (Houbolt 1950) has been used to evaluate the velocity and acceleration terms, $\left(w_{t}\right)_{J}$ and $\left(w_{t t}\right)_{J}$ respectively, in the governing equation of equilibrium.

The expression for velocity and acceleration are

$$
\begin{gathered}
\left(w_{t}\right)_{J}=\left(\alpha_{1} w_{J}+\alpha_{2} w_{J-1}+\alpha_{3} w_{J-2}+\alpha_{4} w_{J-3}+\alpha_{5}\right) /(6 \Delta t), \\
\left(w_{t t}\right)_{J}=\left(\beta_{1} w_{J}+\beta_{2} w_{J-1}+\beta_{3} w_{J-2}+\beta_{4} w_{J-3}+\beta_{5}\right) /\left(6 \Delta t^{2}\right) .
\end{gathered}
$$

Houbolt coefficients $\left(\alpha_{i}, \beta_{i}\right)$ can be evaluated during initial time steps by using recurrence relation and are given in appendix $A$.

Substituting (8) into governing equations (1)-(3), using appropriate boundary conditions (4)-(7) and making use of (9)-(17), gives rise to $3(M+1)(N+1)+24$ linear algebraic equations at each step of the marching variable (loading/time). The total number of unknowns are $3(M+1)(N+1)$. It is seen that the number of equations is more than the number of unknowns. In order to have a compatible and unique solution, multiple regression analysis based on least square error norm is employed. The terms of variable coefficients are transformed to the right hand side so that the left hand side matrix becomes invariant with respect to the marching variable. It is inverted once and kept for subsequent usage. The set of linear algebraic equations can be expressed in matrix form as

$$
\mathbf{A a}=\mathbf{P},
$$


where $A(r \times s)$ is coefficient matrix, $a(s \times 1)$ is an unknown vector and $P(r \times 1)$ is a load vector. Multiple regression analysis yields

$$
\mathbf{a}=\left(\mathbf{A}^{\mathbf{T}} \mathbf{A}\right)^{-1} \mathbf{A}^{\mathbf{T}} \mathbf{P}
$$

or

$$
\mathbf{a}=\mathbf{B P} .
$$

Details are given in appendix $\mathbf{B}$. The matrix $\mathbf{B}$ is evaluated once and stored for subsequent change.

\section{Results and discussions}

In this study, the nonlinear governing equations of equilibrium of isotropic doubly curved shells on rectangular planforms, subjected to uniformly distributed transverse static and dynamic loadings, are considered. The quadratic extrapolation technique is used for linearization of the differential equations. The equations of motion are discretized in space domain using Chebyshev polynomials and the Houbolt scheme is used for the time-domain discretization. By collocating the zeroes of Chebyshev polynomials, more algebraic equations are generated than the number of unknowns and a multiple linear regression analysis based on least-square error norms is employed to solve them. Considering the clamped immovable and simply supported immovable boundary conditions, analyses have been carried out. An increment of 0.5 for non-dimensional load $q^{*}$ and 0.008 for time $t$ are found to yield fairly accurate results.

Convergence studies have revealed that nine terms in the Chebyshev series are sufficient to yield fairly accurate results (table 1). It is seen that the convergence is not monotonic but is of oscillatory nature. Results of static solutions of plates and doubly curved shells have been compared and are shown in tables 2 and 3 respectively. It is clear from these tables that the present results are in good agreement with those obtained by Kanematsu \& Nash (1971) and Kornishin \& Isanbaeva (1968) using finite difference technique.

The variation of central deflection with load for clamped cylindrical panels is plotted in figure 2 for shell parameters $R_{1}=1,2,3,4,8$, respectively. There are many interesting phenomena which are seen in this figure: (i) Shallow panels $\left(R_{1}=1\right.$ and 2) remain stable and their behaviour is of the "hardening" type, (ii) the panel for shell parameter $R_{1}=3$ shows that "snap through" and buckling load is 60.7 , and (iii) the cylindrical panels for $R_{1}=4$ and 5 exhibit softening type behaviour and the buckling loads are 85.5 and 354 respectively.

Table 1. Convergence study for simply supported immovable plates.

\begin{tabular}{lc}
\multicolumn{2}{c}{$\xi=1, R_{1}=R_{2}=0, Q=60$} \\
\hline No. of terms & Max. deflection \\
\hline 7 & 1.205 \\
8 & 1.167 \\
9 & 1.125 \\
10 & 1.139 \\
12 & 1.114 \\
\hline
\end{tabular}


Table 2. Comparison of static results of simply supported immovable plates. $\xi=1.0, R_{1}=R_{2}=0.0$.

\begin{tabular}{lcccc}
\hline Aspect ratio, $\xi$ & Dimensionless load, $Q$ & Present study $w(0) / h^{\mathrm{a}}$ & $w(0) / h$ & $w(0) / h^{\mathrm{b}}$ \\
\hline 1.0 & 29.1 & 0.838 & 0.820 & 0.869 \\
& 65.6 & 1.179 & 1.126 & 1.230 \\
& 147.6 & 1.593 & 1.481 & 1.670 \\
$2 / 3$ & 29.1 & 1.063 & 1.017 & 1.100 \\
& 65.6 & 1.436 & 1.372 & 1.500 \\
\hline
\end{tabular}

References: ${ }^{a}$ Kanematsu \& Nash (1971); ${ }^{b}$ Kornishin \& Isanbaeva (1968)

Table 3. Comparison for doubly curved shells. $\xi=1.0, R_{1}=R_{2}=1.25, Q=19.1$

\begin{tabular}{lcc}
\hline & \multicolumn{2}{c}{ Central deflection } \\
\cline { 2 - 3 } Edge conditions & Present & Kanematsu \\
\hline Clamped immovable & 0.63 & 0.64 \\
Simply supported immovable & 1.44 & 1.64 \\
\hline
\end{tabular}

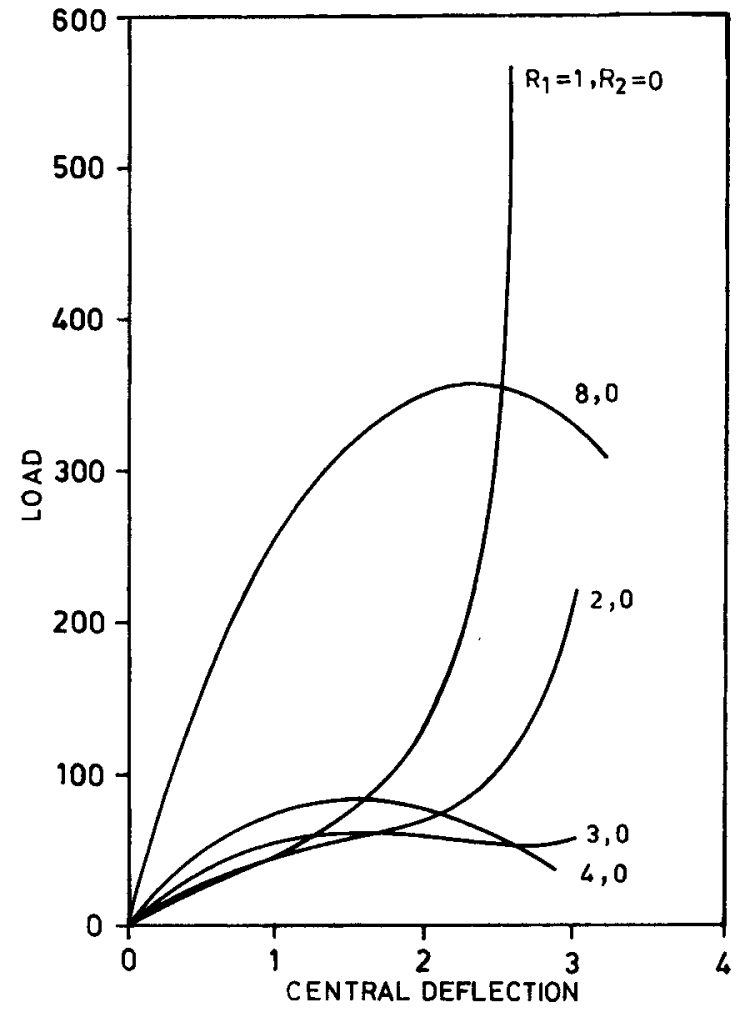

Figure 2. Static deflection of clamped immovable cylindrical panel. 

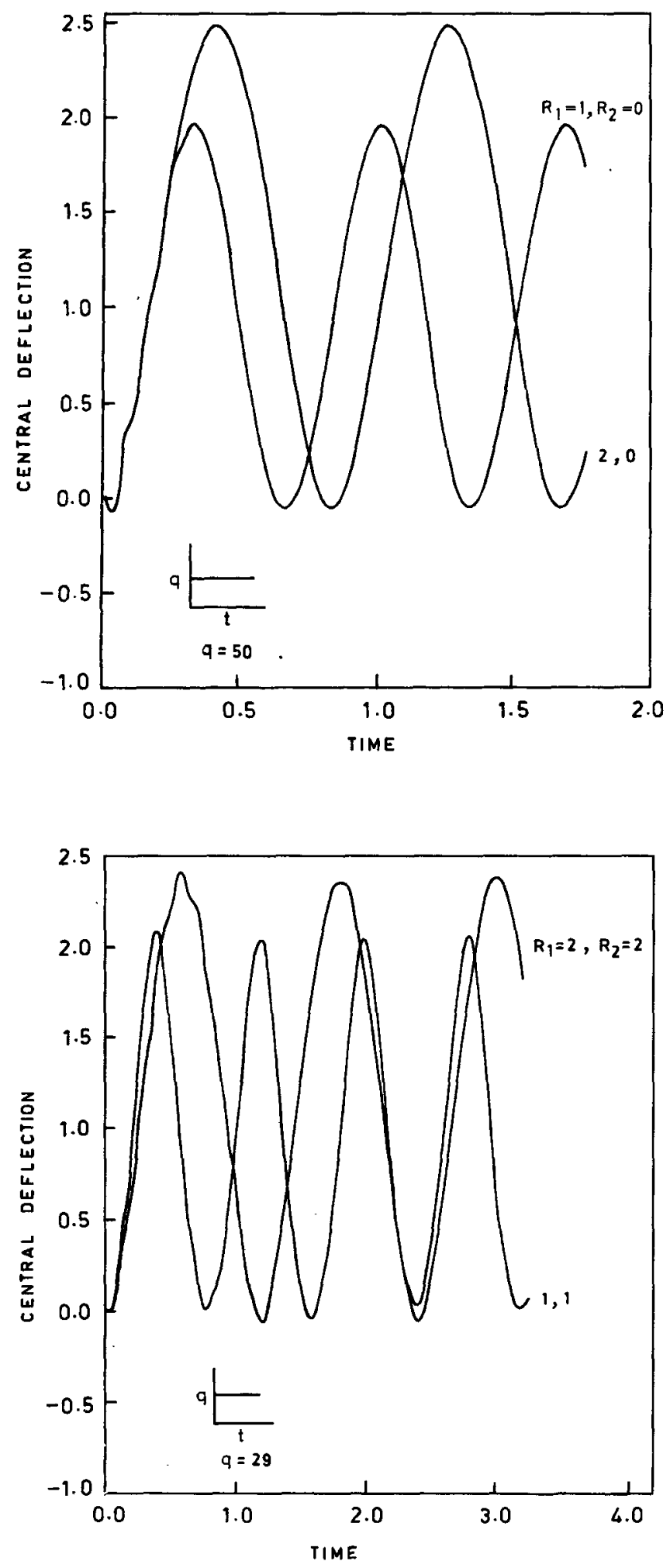

Figure 3. Dynamic response of clamped immovable cylindrical panels due to step loading.

Figure 4. Dynamic response of simply supported immovable doubly curved shells due to step loading. 


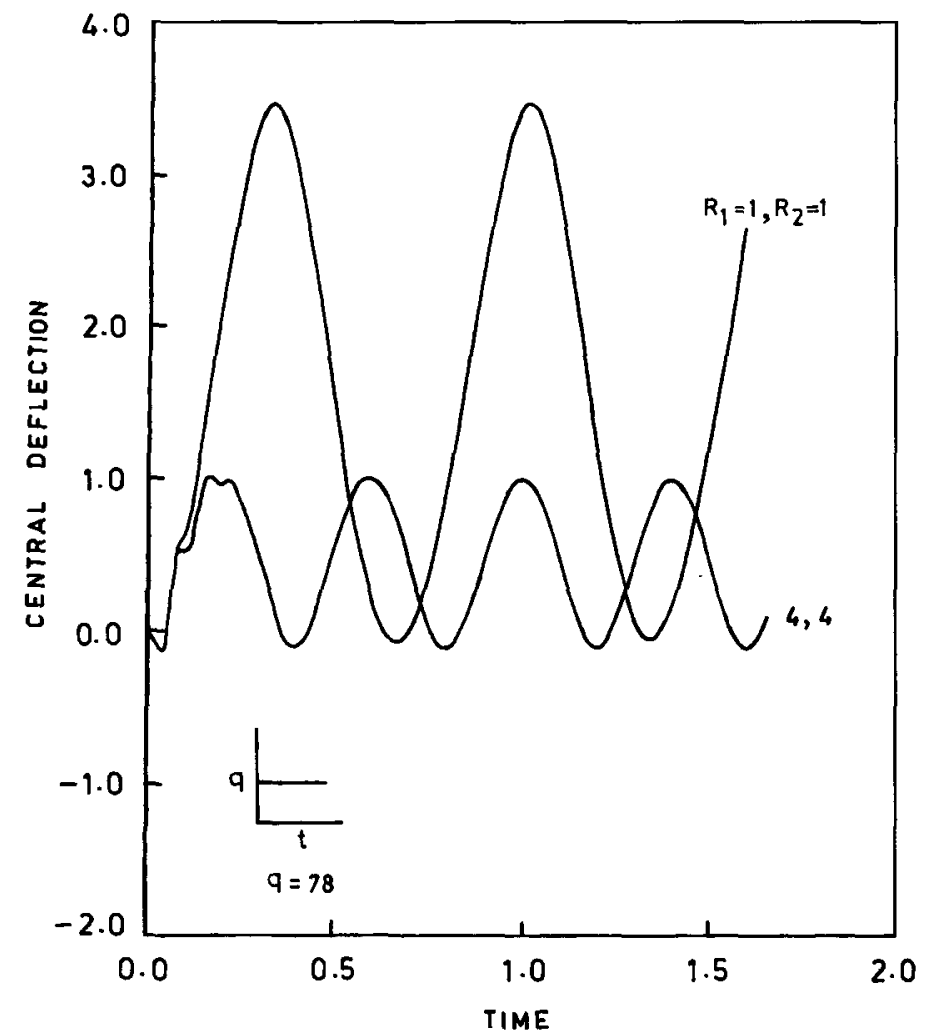

Figure 5. Dynamic response of clamped immovable doubly curved shells due to step loading.

The variation of central deflection with evolution of time for a clamped cylindrical panel is shown in figure 3. The variation of central deflection with time for clamped and simply supported doubly curved shells is shown in figures 4 and 5 respectively. It is clear from these results that the higher the value of shell curvature, the lower the response.

\section{Conclusions}

An analytical approach based on Chebyshev series approximation and nonlinear static and dynamic analysis of doubly curved shells has been presented. The results have been compared with those obtained by other methods. A few typical results are presented. The present methodology is quite efficient and may find applications in other areas.

Part of this work was supported by the Council of Scientific and Industrial Research, New Delhi vide Grant No. 22(276)/97. 
Appendix A. Houbolt coefficients for step-function loading.

\begin{tabular}{|c|c|c|c|c|c|c|c|c|c|c|}
\hline \multirow[b]{2}{*}{ Step } & \multicolumn{5}{|c|}{ Velocity coefficients } & \multicolumn{5}{|c|}{ Acceleration coefficients } \\
\hline & $\alpha_{1}$ & $\alpha_{2}$ & $\alpha_{3}$ & $\alpha_{4}$ & $\alpha_{5}$ & $\beta_{1}$ & $\beta_{2}$ & $\beta_{3}$ & $\beta_{4}$ & $\beta_{5}$ \\
\hline$j=1$ & 18 & 0 & 0 & 0 & $-3 p \Delta \tau$ & 6 & 0 & 0 & 0 & $-2 p \Delta \tau$ \\
\hline$j=2$ & 11 & -16 & 0 & 0 & $-2 p \Delta \tau$ & 2 & -4 & 0 & 0 & $-p \Delta \tau$ \\
\hline$j=3$ & 11 & -18 & 9 & 0 & 0 & 2 & -5 & 4 & 0 & 0 \\
\hline$j>3$ & 11 & -18 & 9 & -2 & 0 & 2 & -5 & 4 & -1 & 0 \\
\hline
\end{tabular}

Appendix B. The algebraic equations can be expressed in matrix form as,

$$
\mathbf{A} \mathbf{a}=\mathbf{P},
$$

where $\mathbf{A}(\mathbf{r} \times \mathbf{s})$ is a coefficient matrix, $\mathbf{a}(\mathbf{s} \times \mathbf{1})$ is a vector and $\mathbf{P}(\mathbf{r} \times \mathbf{1})$ is the load vector. Approximating the solution by introducing the error vector $\mathbf{e}(\mathbf{r} \times \mathbf{1})$, we get

$$
\mathbf{P}=\mathbf{A a}+\mathbf{e} .
$$

To minimize the error norm, let us define a function $\mathbf{S}$ as,

$$
\mathbf{S}(\mathbf{a})=\mathbf{e}^{\mathbf{T}} \mathbf{e}=(\mathbf{P}-\mathbf{A} \mathbf{a})^{\mathbf{T}}(\mathbf{P}-\mathbf{A} \mathbf{a}) .
$$

The least-square norm must satisfy

$$
(\partial \mathbf{S} / \partial \mathbf{a})=-2 \mathbf{a}^{\mathbf{T}} \mathbf{P}+2 \mathbf{A}^{\mathbf{T}} \mathbf{A} \mathbf{a}=\mathbf{0} .
$$

This can be expressed as

$$
\mathbf{a}=\left(\mathbf{A}^{\mathbf{T}} \mathbf{A}\right)^{-1} \mathbf{A}^{\mathbf{T}} \mathbf{P}
$$

or

$$
\mathbf{a}=\mathbf{B P} .
$$

The matrix $\mathbf{B}$ is evaluated once and stored for subsequent use.

\section{List of symbols}

$a, b, h \quad$ dimensions of the shells;

$c^{*}, \gamma, \nu \quad$ damping, density and Poisson's ratio;

$q^{*}, t^{*} \quad$ loading and time;

$R_{1}^{*}, R_{2}^{*} \quad$ principal radii of curvatures;

$u^{*}, v^{*}, w^{*}$ deflection along $x, y$ and $z$ directions.

\section{References}

Alwar R S, Nath Y 1976 Application of Chebyshev polynomials to the nonlinear analysis of circular plates. Int. J. Mech. Sci. 18: 589-595

Chia C Y 1980 Nonlinear analysis of plates (New York: McGraw Hill) 
Evans D J, Murphy C P 1981 The solution of the biharmonic equation in rectangular region by Chebyshev series. Comput. Meth. Appl. Mech. Eng. 27: 81-99

Fox L, Parker I B 1968 Chebyshev polynomials in numerical analysis (Oxford: University Press) Houbolt J C 1950 A recurrence matrix solution for the dynamic response of elastic aircraft. $J$. Aeronaut. Sci. 17: 540-550

Kanematsu H, Nash W A 1971 Random vibration of thin elastic plates and shallow spherical shells. University Massachussetts, AFOSR Scientific Report TR-71-1860

Khedir A A, Reddy J N 1989 On the forced motions of antisymmetric cross ply laminated plates. Int. J. Mech. Sci. 31: 499-510

Kornishin M S, Isanbaeva F S 1968 Flexible plates and panels (Moscow: Izdatelstvo) (in Russian)

Nath Y, Alwar R S 1978 Nonlinear static and dynamic response of spherical shells. Int. J. Nonlinear Mech. 13: 157-170

Nath Y, Sandeep K 1995 Chebyshev series solution to nonlinear boundary value problems in rectangular domain. Comput. Meth. Appl. Mech. Eng. 125: 41-52

Nath Y, Mahrenholtz O, Varma K K 1987 Nonlinear dynamic response of doubly curved shallow shells in elastic foundation. J. Sound Vibr. 112: 53-61 